\title{
Research Progress of Acupuncture and Moxibustion in Treating Constipation After Stroke
}

\author{
Xiaoyu Li1', Sina Feng1, Hui Zhang2* \\ ${ }^{1}$ Shaanxi University of Traditional Chinese Medicine, Xianyang 712046, Shaanxi Province, China \\ ${ }^{2}$ Affiliated Hospital of Shaanxi University of Traditional Chinese Medicine, Xianyang 712046, Shaanxi Province, China \\ *Corresponding author: Hui Zhang, szy19891098038@163.com
}

\begin{abstract}
Constipation is one of the common gastrointestinal complications of stroke, which not only affects the quality of life of patients, but also easily induces and aggravates the primary disease and delays the recovery of the disease. At present, most of the treatments for constipation after stroke are oral medicine and enema, but the curative effect is poor and unstable. Acupuncture has the advantages of simple operation, remarkable effect, high patient acceptance and a little toxic effect. It is one of the non-drug treatment ways to prevent and treat constipation after stroke, as well as widely used in the treatment of constipation after stroke. Therefore, the clinical research on acupuncture treatment of constipation after stroke in recent years is summarized to provide reference for the subsequent clinical treatment of constipation after stroke.
\end{abstract}

Keywords: Acupuncture; After stroke; Constipation; Summarize

Publication date: July 2021; Online publication: July 31, 2021

\section{Introduction}

Stroke is the main clinical type of cerebrovascular disease, which threatens the life and health of middleaged and elderly people. It can cause a variety of complications, among which constipation is one. Studies have shown that the incidence of constipation is about $45 \%$ in patients with acute stroke and about $48 \%$ in rehabilitation period ${ }^{[1]}$. Other data show that when severe limb paralysis occurs in patients with moderate wind, the gastrointestinal function is weakened due to long-term bed rest, and the incidence of constipation is over $90 \%{ }^{[2]}$. At present, the clinical treatment of constipation after stroke is limited to the use of osmotic laxatives, softeners, irritating laxatives or Kaisailu (constipation laxative). Although it has certain curative effect, it has the disadvantages of repeated withdrawal and many side effects ${ }^{[3]}$. In addition, the patients' age is mostly concentrated in middle-aged and elderly, and drug intolerance occurs in most cases, which greatly reduces the curative effect ${ }^{[4]}$. Moreover, if the patient fails to defecate for a long time and the way of defecation is wrong, the intracranial pressure and blood pressure will rise instantly, which will increase the risk of stroke recurrence and cause a vicious circle, thus it is extremely harmful. Due to its unique treatment mechanism, acupuncture treatment can effectively improve the quality of life of patients, also has the advantages of simple operation, economic benefits, obvious effect and less adverse reactions. In this paper, the clinical reports on acupuncture treatment of constipation after stroke in recent years are summarized as follows.

\section{Acupuncture Therapy}

\subsection{Routine acupuncture}

Wang Zhijie et al., ${ }^{[5]}$ found in the treatment of 74 patients with constipation after stroke that the first defecation time and effect duration of acupuncture point group were better than those of lactulose group. 
Similarly, Su Limei et al. ${ }^{[6]}$ treated 37 patients with acupuncture and moxibustion with the method of matching points, by comparing the changes of constipation symptom scores between the two groups, it was found that constipation symptoms in the treatment group improved more obviously. Leng Meng Tong et al., ${ }^{[7]}$ treated 33 patients with constipation after stroke with acupuncture at Shumu points in Weichang, and found that the clinical scores of constipation and fecal traits in the treatment group were lower than those in Liumo Decoction group. Wang Linlin ${ }^{[8]}$ observed the clinical efficacy of 82 patients with constipation after stroke, where the control group was treated with Maren Runchang Pill, while the treatment group was treated with acupuncture. By observing the defecation related indicators of patients, it was found that the first defecation time, each defecation time and defecation interval time of acupuncture group were lower than those of control group, which indicated that acupuncture treatment had considerable effect.

\subsection{Electroacupuncture}

Electroacupuncture treatment of constipation can enhance gastrointestinal peristalsis by enhancing acupuncture effect, and then achieve the effect of defecation. Wang Chengwei ${ }^{[9]}$ and others added electroacupuncture on the basis of routine western medicine, acupuncture treatment and rehabilitation training, and found that electroacupuncture group was better than control group in preventing and treating constipation symptoms. Xiong Zhonghui et al., ${ }^{[10]}$ used abdominal electroacupuncture combined with traditional Chinese medicine application to treat 80 patients with constipation. By comparing the constipation scores of the two groups, it was found that the total effective rate of the treatment group was higher than that of the phenolphthalein tablet group.

\section{Moxibustion}

\subsection{Moxibustion therapy}

Moxibustion therapy achieves the purpose of dredging channels and collaterals and preventing and treating diseases through its warming and dredging effects. Qu Zhimin ${ }^{[11]}$ and others compared the clinical efficacy of wheat moxibustion with ordinary acupuncture, and found that the curative rate of wheat moxibustion group was higher than that of acupuncture group, suggesting that the curative effect of moxibustion was more considerable. Liu Chengmei et al., ${ }^{[12]}$ treated 30 patients with rehabilitation training combined with umbilical moxibustion, and found that constipation in umbilical moxibustion group improved more obviously. Zhang Jiyu ${ }^{[13]}$ treated 40 patients with constipation with heat-sensitive moxibustion on the basis of routine treatment. The results showed that constipation in heat-sensitive moxibustion group was improved more obviously, and the quality of life was improved obviously.

\subsection{Warm acupuncture therapy}

Warm acupuncture is a combined therapy of acupuncture and moxibustion, which has both acupuncture effect and warming and dredging. Lin Guanghua et al., ${ }^{[14]}$ treated 30 patients with quick needling and warm acupuncture, by comparing the scores of constipation symptoms between the two groups, it was found that the total effective rate of warm acupuncture group was higher than that of acupuncture group, and the longterm effect was more obvious. Song Chenyu et al., ${ }^{[15]}$ used warm acupuncture to treat 40 patients with constipation, and found that the total effective rate of warm acupuncture group was significantly higher than that of phenolphthalein tablet group.

\section{Other Therapies}

\subsection{Pressing beans at ear points}

Pressing beans at auricular points plays a therapeutic role by continuously stimulating acupoints. Long Xiaona ${ }^{[16]}$ treated 30 patients with bean pressing at auricular points. By comparing the scores of 
constipation symptoms between the two groups, it was found that the improvement of gastrointestinal symptoms in the treatment group was more significant than that in the Maren Pill group.

\subsection{Catgut embedding at acupoints}

Catgut embedding therapy at acupoints is one of acupuncture methods. Catgut embedding at acupoints can adjust the gastrointestinal tract in a benign and bidirectional way, restore the gastrointestinal function, and effectively treat a variety of gastrointestinal diseases. Du Jia ${ }^{[17]}$ and others used acupoint catgut embedding method to treat 105 patients with constipation, and found that the number of defecation, the difficulty of defecation and the improvement of fecal characteristics were better than those of the control group. Susan Wang ${ }^{[18]}$ treated 30 patients with catgut embedding at acupoints, and found that the improvement of constipation symptom score was better than that of conventional acupuncture.

\subsection{Acupoint application}

By applying the medicine to specific acupoints, the medicine can directly penetrate into the skin and follow the meridians, thus improving constipation. Chen Weiwei et al., ${ }^{[19]}$ applied raw rhubarb powder to acupoints on the basis of routine nursing, and the results showed that the total effective rate of the treatment group was higher than that of the control group.

\subsection{Acupoint massage}

Acupoint massage directly focuses on the abdomen or corresponding acupoints through massage techniques, changes gastrointestinal morphology, accelerates gastrointestinal peristalsis, and stimulates acupoints to play the role of dredging meridians, regulating abdominal qi and blood, promoting stool discharge and reducing constipation ${ }^{[20]}$. Liang Binglian ${ }^{[21]}$ applied routine nursing and acupoint massage to 50 patients with constipation, and found that acupoint massage can effectively improve constipation symptoms.

\section{Discussion}

To sum up, through the research of acupuncture, pressing beans at ear points, embedding thread at acupoints, applying and massaging, is found that traditional Chinese medicine therapy can effectively improve clinical symptoms, relieve illness and improve patients' life satisfaction. Moreover, the traditional therapy has no dependence and high patient compliance, which can improve the gastrointestinal function of patients and achieve the purpose of treating both the symptoms and root causes, showing that the traditional therapy has irreplaceable advantages in this field. However, there are still many clinical problems, such as the mechanism of acupuncture treatment is not completely clear, which needs further discussion and research; Acupuncture and moxibustion treatment methods are complicated, and the best treatment scheme for diseases in different periods cannot be put forward clinically, which still needs to be explored and studied; Acupuncture has good short-term effect, but poor long-term effect, which cannot solve the problem fundamentally, thus it still needs further exploration.

\section{Disclosure statement}

The author declares no conflict of interest.

\section{References}

[1] Li J, Yuan M, Liu Y, et al., 2017, Incidence of Constipation in Stroke Patients: A Systematic Review and Meta-Analysis, Medicine (Baltimore), 96(25): e7225.

[2] Peng G, Mi Y, 2019, Research Progress of Acupuncture Treatment of Constipation After Stroke, 
Xinjiang traditional Chinese medicine, 37(01):137-140.

[3] Liu S, Liao X, Jia M, et al., 2020, Systematic Evaluation of the Efficacy and Safety of Acupuncture in Treating Constipation After Stroke, Beijing Traditional Chinese Medicine, 39(11):1135-1139.

[4] Dong C, Gao Y, Li Y, et al., 2021, Observation on the Therapeutic Effect of Acupuncture on Constipation After Ischemic Stroke, Inner Mongolia Traditional Chinese Medicine, 40(01):104105.

[5] Wang Z, Liu C, Min Y, et al., 2021, Treatment of Constipation After Stroke with Acupuncture at Odd Points Combined with Syndrome Differentiation, Jilin Traditional Chinese Medicine, 41(01):119-122.

[6] Su L, Pan J, Zeng K, 2018, Analysis of Therapeutic Effect of Acupuncture and Moxibustion Combined with Acupoints on Constipation After Stroke, Shenzhen Journal of Integrative Medicine, 28(12):53-54.

[7] Leng M, Wang J, Liu T, et al., 2019, Randomized Controlled Study of Acupuncture at Shumu Point in Gastrointestinal Tract on Constipation After Stroke, Shanghai Journal of Acupuncture, 38(02):178-182.

[8] Wang L, 2020, Observation on Therapeutic Effect of Acupuncture on Constipation After Ischemic Stroke, Guide of China Medicine, 18(10):185-186.

[9] Wang C, Liu M, Wen Q, et al, 2015, Clinical Observation on Electroacupuncture Intervention in Prevention and Treatment of Constipation in Acute Stage of Ischemic Stroke, Chinese Acupuncture, 35(05):430-434.

[10] Xiong Z, Gong X, Lu T, 2017, Observation on the Therapeutic Effect of Abdominal Electroacupuncture Combined with Umbilical Application of Traditional Chinese Medicine on Constipation After Stroke, Shanghai Journal of Acupuncture and Moxibustion, 36(03):265-268.

[11] Qu Z, Ji Y, Wang S, et al., 2020, Comparison of Therapeutic Effects of Wheat Grain Moxibustion and Ordinary Acupuncture on Constipation in Patients with Ischemic Stroke, Clinical Journal of Traditional Chinese Medicine, 32(02):313-316.

[12] Liu C, Feng X, Liu F, et al., 2015, Effect of Umbilical Moxibustion on Constipation After Stroke, Chinese Rehabilitation Theory and Practice, 21(10):1209-1211.

[13] Zhang J, 2018, Clinical Analysis of Heat-Sensitive Moxibustion in Treating Constipation After Stroke, Chinese and Foreign Medical Research, 16(05):31-33.

[14] Lin G, Zhao B, 2016, Observation on the Therapeutic Effect of Acupuncture and Moxibustion on Constipation After Stroke, New Traditional Chinese Medicine, 48(07):43-44.

[15] Song C, Liu L, 2015, Observation on Therapeutic Effect of Warm Acupuncture on Constipation After Stroke, Everybody Health (Academic Edition), 9(16):40.

[16] Long X, Liu L, 2017, Observation on the Therapeutic Effect of Bean Implantation at Ear Points on Constipation After Stroke, Journal of Anhui University of Traditional Chinese Medicine, 36(03):50-52.

[17] Du J, Liu H, Xu J, 2020, Catgut Embedding Therapy for Constipation After Stroke: A Multicenter Randomized Controlled Study, Chinese Acupuncture, 40(05):493-497.

[18] Wang S, Fan M, 2017, A Randomized Parallel Controlled Study of Catgut Embedding at Acupoints Combined with Western Medicine in Treating Constipation After Stroke, Journal of Practical Traditional Chinese Internal Medicine, 31(07):32-35. 
[19] Chen W, Gao C, 2019, Observation on Curative Effect and Nursing Care of 80 Cases of Constipation After Stroke Treated by Acupoint Application of Raw Rhubarb Powder, Journal of Psychology, 14(21):155.

[20] Ma C, Yin L, Tian X, Li C, 2019, Research Survey of Acupuncture Treatment of Constipation After Stroke, Journal of Traditional Chinese Medicine, Hunan, 35(01):141-144.

[21] Liang B, Feng L, Sharla C, et al., 2020, Observation on Nursing Effect of "Fu Yuan Tong Fu Fu" Abdominal Massage on Constipation After Stroke, Journal of Clinical Nursing, 19(03):79-81. 